Check for updates

Cite this: RSC Adv., 2019, 9, 38797

\title{
Nafion film transport properties in a low-Pt PEM fuel cell: impedance spectroscopy study
}

\author{
Tatyana Reshetenko (D) a and Andrei Kulikovsky (D) *b \\ Unexpected over-linear transport loss in low-Pt PEM fuel cells has been a subject of numerous studies and \\ discussions in literature. Most of the authors agree that these losses are due to oxygen transport in the \\ Nafion film covering Pt/C agglomerates in the cathode catalyst layer (CCL). We develop a model for PEM \\ fuel cell impedance, which takes into account oxygen transport through the film. The model is fitted to \\ experimental impedance spectra of a low-Pt PEM fuel cell. Fitting gives the film thickness in the range of \\ 10 to $13 \mathrm{~nm}$, and the film transport resistivity decreasing from $1.2 \mathrm{~s} \mathrm{~cm}^{-1}$ to $0.2 \mathrm{~s} \mathrm{~cm}^{-1}$ as the cell current \\ density increases from 50 to $800 \mathrm{~mA} \mathrm{~cm}{ }^{-2}$. Fitting returns low value of the through-plane oxygen \\ diffusivity in the $C C L$, indicating that the low-Pt electrode is partially flooded.
}

Received 25th September 2019 Accepted 7th November 2019

DOI: $10.1039 / c 9 r a 07794 d$

rsc.li/rsc-advances resistivity is due to $\mathrm{Pt} /$ ionomer interface. More discussions of the problem and references can be found in recent reviews. ${ }^{7,8}$

An alternative explanation of poor performance of low-Pt cathodes has been suggested by Muzaffar $e t$ al. ${ }^{9}$ They processed numerous experimental polarization curves and showed that the thin, low-Pt electrodes are prone to flooding. Essentially, the effect is due to much lower surface of liquid water in a thin electrode, which strongly reduces its water evaporation ability. ${ }^{10}$

In this work, we report a single-pore transient model for the CCL performance, which includes oxygen transport through the Nafion film. A static version of the model has been analyzed in ref. 11. In the limit of fast proton and through-plane oxygen transport in the CCL, analytical solution for the CCL performance has been derived. The solution reveals the limiting current density due to oxygen transport through the Nafion film; moreover, this limiting current appears to be proportional to the CCL thickness. ${ }^{11}$ This result may explain a strong effect of the Nafion film on performance of low-Pt electrodes, as these electrodes are three to four times thinner, than the standard systems.

Below, the transient model is used for construction of numerical impedance model of a cell. Fitting of model impedance to experimental spectra of the low-Pt cell shows that the Nafion film thickness varies in the range of 10 to $13 \mathrm{~nm}$, while the Nafion film transport resistance decreases with the cell current. To the best of our knowledge, this result is a first measurement of Nafion film thickness and transport parameter in an operating low-Pt PEM fuel cell. At low currents, the though-plane oxygen diffusion coefficient in the electrode appears to be close to the oxygen diffusivity in liquid water, indicating that the low-Pt cathode is partially flooded. It is worth noting that with minor modifications, the model below can be used for processing the spectra of electrochemical power sources utilizing thin electrolyte films over active materials (e.g., in supercapacitors, ${ }^{12}$ or Li-ion batteries ${ }^{13}$ ).
${ }^{a}$ Hawaii Natural Energy Institute, University of Hawaii, Honolulu, Hawaii 96822, USA. E-mail: tatyanar@hawaii.edu

${ }^{b}$ Forschungszentrum Jülich $\mathrm{GmbH}$, Institute of Energy and Climate Research, IEK-14: Electrochemical Process Engineering, D-52425 Jülich, Germany. E-mail: A. Kulikovsky@fz-juelich.de 


\section{Experimental}

Spatial electrochemical impedance spectroscopy (EIS) recording and measurements of localized current density and voltage have been performed using a segmented cell system and a test station developed at Hawaii Natural Energy Institute. The segmented cell setup consists of the cell hardware, the custom designed current transducer system, the data acquisition device and a single cell test station. Details of the segmented cell system can be found in our previous publications. ${ }^{\mathbf{1 4 , 1 5}}$

The segmented cell hardware design is based on $100 \mathrm{~cm}^{2}$ cell and consists of non-segmented and segmented flow field plates. Both flow field plates have the same ten parallel channel serpentine design and arranged in co-flow configuration. The segmented plate consists of 10 segments with an area of $7.6 \mathrm{~cm}^{2}$ each arranged in two parallel rows from inlet (segment 1) to outlet (segment 10). Each segment has its own current collector and gas diffusion layer (GDL). The segmentation was applied to the cathode.

In this work, Gore catalyst coated membranes (CCM) with the active area of $100 \mathrm{~cm}^{2}$ were used. Catalyst loading was 0.1 $\mathrm{mg}_{\mathrm{Pt}} \mathrm{cm}^{-2}$ for anode and cathode with the catalyst layer thickness of 3-4 $\mu \mathrm{m}$. The thickness of reinforced membrane varied in the range of $16-18 \mu \mathrm{m}$. Sigracet $25 \mathrm{BC}$ was used as GDLs for both electrodes. 25BC consists of carbon paper and microporous layer (MPL) with total thickness of 220-235 $\mu \mathrm{m}$ and porosity of $80 \%$. Segmented GDLs were used on the cathode side, whereas a single and uniform GDL was applied at the anode. Teflon gaskets $(125 \mu \mathrm{m})$ were employed for both electrodes to ensure required compression ratio.

The cell was operated with $\mathrm{H}_{2}$ /air gas configuration at a temperature of $80{ }^{\circ} \mathrm{C}$. The anode/cathode conditions were $2 / 4$ stoichiometry, $100 \% / 50 \%$ relative humidity and 150/150 $\mathrm{kPa}$ backpressure. The EIS curves were recorded under galvanostatic control of the overall cell over the frequency range from $0.05 \mathrm{~Hz}$ to $10 \mathrm{kHz}$ with 11 steps per decade. The amplitude of sinusoidal current perturbation signal was chosen to provide a cell voltage response of $10 \mathrm{mV}$ or lower. EIS were recorded simultaneously from 10 segments and from the whole cell. In this work, we have processed only the whole cell spectra using, however, measured values of the local current density in impedance calculations.

\section{Model}

\subsection{Transient performance equations}

SEM pictures show that pores in real cathode catalyst layers (CCLs) are formed by numerous agglomerates of Pt/C particles, surrounded by a thin Nafion film. ${ }^{16}$ An idealized straight single pore penetrating through the whole CCL depth is shown schematically in Fig. 1a. We will model this system by cylindrical pore of the radius $R_{\mathrm{p}}$ with the thin Nafion film separating the void pore volume from the cylindrical Pt wall of the radius $R_{\mathrm{m}}$ (Fig. 1b).

The model below is a transient extension of the static model for polarization curve of the system in Fig. 1b. Due to strong electrochemical coupling of potential, current and oxygen concentration in a fuel cell, we first need a time-dependent
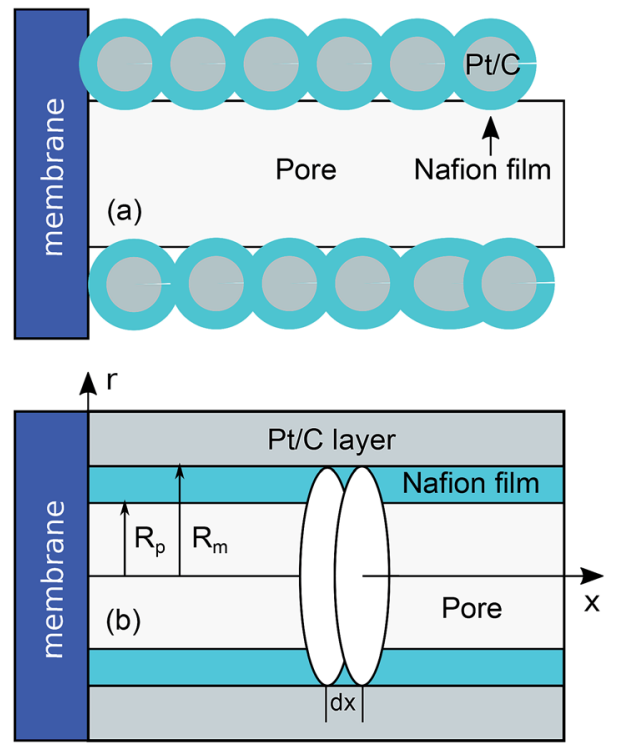

Fig. 1 (a) Schematic of a single pore in the CCL, (b) the system for modeling.

oxygen mass transport equation in the pore volume. Consider cylindrical element of the pore/Nafion film volume of the radius $R_{\mathrm{m}}$ and the thickness $\mathrm{d} x$ (Fig. 1b). The oxygen diffusive flux along the pore is $D_{\mathrm{p}} \partial c / \partial x$, where $D_{\mathrm{p}}$ is the effective oxygen diffusion coefficient, and $c$ is the local oxygen concentration in the pore. The balance of fluxes in the pore element leads to

$$
-\left.\pi R_{\mathrm{p}}^{2} D_{\mathrm{p}} \frac{\partial c}{\partial x}\right|_{x+\mathrm{d} x}+\left.\pi R_{\mathrm{p}}^{2} D_{\mathrm{p}} \frac{\partial c}{\partial x}\right|_{x}=\left(2 \pi R_{\mathrm{m}} \mathrm{d} x\right) N_{\mathrm{N}, \mathrm{m}}
$$

where

$$
N_{\mathrm{N}, \mathrm{m}}=\left.D_{\mathrm{N}} \frac{\partial c_{\mathrm{N}}}{\partial r}\right|_{r=R_{\mathrm{m}}}
$$

is the radial oxygen flux at the metal/Nafion interface consumed in the ORR, $c_{N}$ is the dissolved oxygen concentration in the Nafion film and $D_{\mathrm{N}}$ is the effective oxygen diffusivity in the film.

Dividing both sides of eqn (1) by $\pi R_{\mathrm{p}}{ }^{2} \mathrm{~d} x$, we come to

$$
-D_{\mathrm{p}} \frac{\partial^{2} c}{\partial x^{2}}=\frac{2 R_{\mathrm{m}} N_{\mathrm{N}, \mathrm{m}}}{R_{\mathrm{p}}^{2}}
$$

The transient version of eqn (3) reads

$$
\frac{\partial c}{\partial t}-D_{\mathrm{p}} \frac{\partial^{2} c}{\partial x^{2}}=\frac{2 R_{\mathrm{m}} N_{\mathrm{N}, \mathrm{m}}}{R_{\mathrm{p}}{ }^{2}},\left.\quad \frac{\partial c}{\partial x}\right|_{x=0}=0, \quad c\left(l_{\mathrm{t}}\right)=c_{1},
$$

where $c_{1}$ is the oxygen concentration at the CCL/GDL interface, and $l_{\mathrm{t}}$ is the CCL thickness (the pore length).

Oxygen transport through the Nafion film along the radial direction is described by

$$
\begin{gathered}
\frac{\partial c_{\mathrm{N}}}{\partial t}-\frac{D_{\mathrm{N}}}{r} \frac{\partial}{\partial r}\left(r \frac{\partial c_{\mathrm{N}}}{\partial r}\right)=0, \quad c_{\mathrm{N}}\left(R_{\mathrm{p}}\right)=K_{\mathrm{H}} c(x), \\
\left.D_{\mathrm{N}} \frac{\partial c_{\mathrm{N}}}{\partial r}\right|_{r=R_{\mathrm{m}}}=-\frac{i * R_{\mathrm{p}}^{2}}{2 R_{\mathrm{m}}(4 F)}\left(\frac{c_{\mathrm{N}, \mathrm{m}}}{c_{\mathrm{h}}^{\text {in }}}\right) \exp \left(\frac{\eta}{b}\right)
\end{gathered}
$$


Here, $K_{\mathrm{H}}$ is the dimensionless Henry constant $\left(\mathrm{mol} \mathrm{mol}^{-1}\right)$ for oxygen solubility in Nafion, $c_{\mathrm{N}, \mathrm{m}} \equiv c_{\mathrm{N}}\left(R_{\mathrm{m}}\right), c_{\mathrm{h}}^{\text {in }}$ is the reference (inlet) oxygen concentration, $b$ is the ORR Tafel slope, and $i_{*}$ is the ORR exchange current density. The first boundary condition in eqn (5) is obvious, while the second means that the oxygen flux at the Nafion/Pt interface equals the flux consumed in the ORR at the Pt surface. This flux is given by the product of the Tafel ORR rate

$$
\frac{i_{*}}{4 F}\left(\frac{c_{\mathrm{N}, \mathrm{m}}}{c_{\mathrm{h}}^{\text {in }}}\right) \exp \left(\frac{\eta}{b}\right)
$$

by the characteristic length $R_{\mathrm{p}}{ }^{2} /\left(2 R_{\mathrm{m}}\right)$. This boundary condition provides correct transition to the limiting case of vanishing Nafion film. Indeed, setting $K_{\mathrm{H}}=1, R_{\mathrm{p}}=R_{\mathrm{M}}$, we may omit eqn (5), set $c_{\mathrm{N}}=c$ and substitute the flux on the right side of the second boundary condition in eqn (5) instead of $N_{\mathrm{N}, \mathrm{m}}$ into eqn (4). This gives

$$
\frac{\partial c}{\partial t}-D_{\mathrm{p}} \frac{\partial^{2} c}{\partial x^{2}}=-\frac{i_{*}}{4 F}\left(\frac{c}{c_{\mathrm{h}}^{\text {in }}}\right) \exp \left(\frac{\eta}{b}\right)
$$

which is a standard macrohomogeneous model (MHM) equation for oxygen transport in the CCL.

The system of eqn (4) and (5) is completed by proton current conservation equation in the Nafion film:

$$
C_{\mathrm{dl}} \frac{\partial \eta}{\partial t}+\frac{\partial j}{\partial x}=-i *\left(\frac{c_{\mathrm{N}, \mathrm{m}}}{c_{\mathrm{h}}^{\text {in }}}\right) \exp \left(\frac{\eta}{b}\right) .
$$

where $C_{\mathrm{dl}}$ is the double layer capacitance. Using the Ohm's law

$$
j=-\sigma_{\mathrm{N}} \frac{\partial \eta}{\partial x}
$$

to eliminate $j$ from eqn (7), we get

$$
C_{\mathrm{dl}} \frac{\partial \eta}{\partial t}-\sigma_{\mathrm{N}} \frac{\partial^{2} \eta}{\partial x^{2}}=-i *\left(\frac{c_{\mathrm{N}, \mathrm{m}}}{c_{\mathrm{h}}^{\text {in }}}\right) \exp \left(\frac{\eta}{b}\right)
$$

where $\sigma_{\mathrm{N}}$ is the Nafion film proton conductivity.

To simplify calculations we introduce dimensionless variables

$$
\tilde{t}=\frac{t}{t_{*}}, \quad \tilde{x}=\frac{x}{l_{\mathrm{t}}}, \quad \tilde{j}=\frac{j}{j_{\mathrm{p}}}, \quad \tilde{\eta}=\frac{\eta}{b}, \quad \tilde{r}=\frac{r}{l_{\mathrm{t}}}
$$

where

$$
t_{*}=\frac{C_{\mathrm{dl}} b}{i_{*}}, \quad j_{\mathrm{p}}=\frac{\sigma_{\mathrm{N}} b}{l_{\mathrm{t}}}
$$

are the characteristic time of double layer charging and current density for proton transport, respectively. With this, eqn (4), (5) and (9) transform to

$$
\begin{gathered}
\mu^{2} \frac{\partial \tilde{c}}{\partial \tilde{t}}-\varepsilon_{*}^{2} \tilde{D}_{\mathrm{p}} \frac{\partial^{2} \tilde{c}}{\partial \tilde{x}^{2}}=\varepsilon \varepsilon_{*}^{2} \tilde{N}_{\mathrm{N}, \mathrm{m}},\left.\quad \frac{\partial \tilde{c}}{\partial \tilde{x}}\right|_{\tilde{x}=0}=0, \quad \tilde{c}(1)=\tilde{c}_{1}, \\
\mu^{2} \frac{\partial \tilde{c}_{\mathrm{N}}}{\partial \tilde{t}}-\varepsilon_{*}^{2} \tilde{D}_{\mathrm{N}} \frac{1}{\tilde{r}} \frac{\partial}{\partial \tilde{r}}\left(\tilde{r} \frac{\partial \tilde{c}_{\mathrm{N}}}{\partial \tilde{r}}\right)=0, \quad \tilde{c}_{\mathrm{N}, \mathrm{p}}=K_{\mathrm{H}} \tilde{c}(\tilde{x}), \\
\left.\varepsilon \varepsilon_{*}^{2} \tilde{D}_{\mathrm{N}} \frac{\partial \tilde{c}_{\mathrm{N}}}{\partial \tilde{r}}\right|_{\tilde{r}=\tilde{R}_{\mathrm{m}}}=-\tilde{c}_{\mathrm{N}, \mathrm{m}} \exp \tilde{\eta}
\end{gathered}
$$

$$
\frac{\partial \tilde{\eta}}{\partial \tilde{t}}-\varepsilon_{*}^{2} \frac{\partial^{2} \tilde{\eta}}{\partial \tilde{x}^{2}}=-\tilde{c}_{\mathrm{N}, \mathrm{m}} \exp \tilde{\eta},
$$

where $\tilde{c}_{\mathrm{N}, \mathrm{p}} \equiv \tilde{c}_{\mathrm{N}}\left(\tilde{R}_{\mathrm{p}}\right)$. In eqn (12), the dimensionless flux $\tilde{N}_{\mathrm{N}, \mathrm{m}}$ is given by

$$
\tilde{N}_{\mathrm{N}, \mathrm{m}}=\frac{4 F l_{\mathrm{t}} N_{\mathrm{N}, \mathrm{m}}}{\sigma_{\mathrm{N}} b}=\left.\tilde{D}_{\mathrm{N}} \frac{\partial \tilde{c}_{\mathrm{N}}}{\partial \tilde{r}}\right|_{\tilde{r}=\tilde{R}_{\mathrm{m}}},
$$

the oxygen diffusion coefficients are normalized according to

$$
\tilde{D}=\frac{4 F D c_{\mathrm{h}}^{\text {in }}}{\sigma_{\mathrm{N}} b}
$$

and $\varepsilon, \mu$ and $\varepsilon *$ are the dimensionless parameters

$$
\varepsilon=\frac{2 \tilde{R}_{\mathrm{m}}}{\tilde{R}_{\mathrm{p}}^{2}}, \quad \mu=\sqrt{\frac{4 F c_{\mathrm{h}}^{\mathrm{in}}}{C_{\mathrm{dl}} b}}, \quad \varepsilon *=\sqrt{\frac{\sigma_{\mathrm{N}} b}{i_{*} l_{\mathrm{t}}^{2}}}
$$

\subsection{Static equations}

For further references, we need a system of static equations for $\tilde{c}^{0}, \tilde{\eta}^{0}$ and $\tilde{j}^{0}$. Here, the superscript 0 marks the static variables. The procedure of the static equations derivation is described in ref. 11; for completeness, this procedure is reproduced in Appendix. The resulting system of equations for $\tilde{c}^{0}$ and $\tilde{j}^{0}$ is

$$
\begin{gathered}
\frac{\partial^{2} \tilde{j}^{0}}{\partial \tilde{x}^{2}}-\left\{\frac{\partial \ln \left(\tilde{c}^{0}\right)}{\partial \tilde{x}}-\left(1+\frac{\alpha \varepsilon_{*}^{2}}{K_{\mathrm{H}} \tilde{c}^{0}} \frac{\partial \tilde{j}^{0}}{\partial \tilde{x}}\right) \tilde{j}^{0}\right\} \frac{\partial \tilde{j}^{0}}{\partial \tilde{x}}=0, \\
\tilde{j}^{0}(0)=\tilde{j}^{0}, \quad \tilde{j}^{0}(1)=0 \\
\tilde{D}_{\mathrm{p}} \frac{\partial \tilde{c}^{0}}{\partial \tilde{x}}=\tilde{j}_{0}-\tilde{j}^{0}, \quad \tilde{c}^{0}(1)=\tilde{c}_{1},
\end{gathered}
$$

where $\alpha>0$ is a constant parameter:

$$
\alpha=\frac{\tilde{R}_{\mathrm{m}}}{\varepsilon \varepsilon_{*}^{2} \tilde{D}_{\mathrm{N}}} \ln \left(\frac{R_{\mathrm{m}}}{R_{\mathrm{p}}}\right) .
$$

With $\tilde{c}^{0}(\tilde{x})$ and $\tilde{j}^{0}(\tilde{x})$ at hand, the static overpotential $\tilde{\eta}^{0}(\tilde{x})$ is obtained from

$$
\tilde{\eta}^{0}=\ln \left(-\frac{\varepsilon_{*}^{2} \partial \hat{j}^{0} / \partial \tilde{x}}{K_{\mathrm{H}} \tilde{c}^{0}+\alpha \varepsilon_{*}^{2} \partial \hat{j}^{0} / \partial \tilde{x}}\right) .
$$

\subsection{Equations for the perturbation amplitudes}

Now we can excite the system with small-amplitude harmonic perturbations of the form

$$
\tilde{c}(\tilde{x}, \tilde{t})=\tilde{c}^{0}(\tilde{x})+\tilde{c}^{1}(\tilde{x}, \tilde{\omega}) \exp (\mathrm{i} \tilde{\omega} \tilde{t}), \quad \tilde{c}^{1} \ll \tilde{c}^{0}
$$

and similar for $\tilde{c}_{\mathrm{N}}$ and $\tilde{\eta}$. Here, the superscript 1 denotes perturbation amplitudes, $\tilde{\omega}=\omega t_{*}$, and $\omega$ is the angular frequency of the AC signal. Substituting these Fourier-transforms into eqn (12)-(14), subtracting the respective static equations, expanding exponent in eqn (14) over small $\tilde{\eta}^{1}$ and neglecting the term with perturbation product, we get a system 
of linear equations for the perturbation amplitudes in the $\omega$ space:

$$
\begin{gathered}
\varepsilon_{*}^{2} \tilde{D}_{\mathrm{p}} \frac{\partial^{2} \tilde{c}^{1}}{\partial \tilde{x}^{2}}=-\left.\varepsilon \varepsilon_{*}^{2} \tilde{D}_{\mathrm{N}} \frac{\partial \tilde{c}_{\mathrm{N}}^{1}}{\partial \tilde{r}}\right|_{\tilde{r}=\tilde{R}_{\mathrm{m}}}+\mathrm{i} \tilde{\omega} \mu^{2} \tilde{c}^{1}, \\
\left.\frac{\partial \tilde{c}^{1}}{\partial \tilde{x}}\right|_{\tilde{x}=0}=0, \quad \tilde{c}^{1}(1)=\tilde{c}_{\mathrm{b}}^{1}(1) \\
\varepsilon_{*}^{2} \tilde{D}_{\mathrm{N}} \frac{1}{\tilde{r}} \frac{\partial}{\partial \tilde{r}}\left(\tilde{r} \frac{\partial \tilde{c}_{\mathrm{N}}^{1}}{\partial \tilde{r}}\right)=\mathrm{i} \tilde{\omega} \mu^{2} \tilde{c}_{\mathrm{N}}^{1}, \quad \tilde{c}_{\mathrm{N}}^{1}\left(\tilde{R}_{\mathrm{p}}\right)=K_{\mathrm{H}} \tilde{c}^{1}(\tilde{x}), \\
\left.\varepsilon \varepsilon_{*}^{2} \tilde{D}_{\mathrm{N}} \frac{\partial \tilde{c}_{\mathrm{N}}^{1}}{\partial \tilde{r}}\right|_{\tilde{r}=\tilde{R}_{\mathrm{m}}}=-\exp \left(\tilde{\eta}^{0}\right)\left(\tilde{c}_{\mathrm{N}, \mathrm{m}}^{1}+\tilde{c}_{\mathrm{N}, \mathrm{m}}^{0} \tilde{\eta}^{1}\right) \\
\varepsilon_{*}^{2} \frac{\partial^{2} \tilde{\eta}^{1}}{\partial \tilde{x}^{2}}=\exp \left(\tilde{\eta}^{0}\right)\left(\tilde{c}_{\mathrm{N}, \mathrm{m}}^{1}+\tilde{c}_{\mathrm{N}, \mathrm{m}}^{0} \tilde{\eta}^{1}\right)+\mathrm{i} \tilde{\omega} \tilde{\eta}^{1}, \\
\tilde{\eta}^{1}(1)=\tilde{\eta}_{1}^{1},\left.\quad \frac{\partial \tilde{\eta}^{1}}{\partial \tilde{x}}\right|_{\tilde{x}=1}=0 .
\end{gathered}
$$

The right boundary condition in eqn (23) describes continuity of the oxygen concentration perturbation at the CCL/GDL interface. Here, $\tilde{c}_{\mathrm{b}}^{1}$ is the perturbation amplitude of the oxygen concentration in the GDL, which is determined from the solution of oxygen transport in the GDL (see below).

Taking into account the second boundary condition to eqn (24), eqn (23) transforms to

$$
\begin{gathered}
\varepsilon_{*}^{2} \tilde{D}_{\mathrm{p}} \frac{\partial^{2} \tilde{c}^{1}}{\partial \tilde{x}^{2}}=\exp \left(\tilde{\eta}^{0}\right)\left(\tilde{c}_{\mathrm{N}, \mathrm{m}}^{1}+\tilde{c}_{\mathrm{N}, \mathrm{m}}^{0} \tilde{\eta}^{1}\right)+\mathrm{i} \tilde{\omega} \mu^{2} \tilde{c}^{1}, \\
\left.\frac{\partial \tilde{c}^{1}}{\partial \tilde{x}}\right|_{\tilde{x}=0}=0, \quad \tilde{c}^{1}(1)=\tilde{c}_{\mathrm{b}}^{1}(1)
\end{gathered}
$$

The system (24)-(26) can be simplified. Solving eqn (24) and setting $\tilde{r}=\tilde{R}_{\mathrm{m}}$ in the solution we get $\tilde{c}_{\mathrm{N}, \mathrm{m}}^{1}$, which appears in eqn (25) and (26):

$$
\tilde{c}_{\mathrm{N}, \mathrm{m}}^{1}=\frac{1}{B}\left(-q A_{c} K_{\mathrm{H}} \tilde{c}^{1}+A_{\eta} E \tilde{c}_{\mathrm{N}, \mathrm{m}}^{0} \tilde{\eta}^{1}\right)
$$

where

$$
\begin{aligned}
A_{c}=\mathrm{i} J_{0}\left(q \tilde{R}_{\mathrm{m}}\right) K_{1}\left(-\mathrm{i} q \tilde{R}_{\mathrm{m}}\right)+J_{1}\left(q \tilde{R}_{\mathrm{m}}\right) K_{0}\left(-\mathrm{i} q \tilde{R}_{\mathrm{m}}\right) \\
A_{\eta}=J_{0}\left(q \tilde{R}_{\mathrm{p}}\right) K_{0}\left(-\mathrm{i} q \tilde{R}_{\mathrm{m}}\right)-J_{0}\left(q \tilde{R}_{\mathrm{m}}\right) K_{0}\left(-\mathrm{i} q \tilde{R}_{\mathrm{p}}\right) \\
B=\left(J_{0}\left(q \tilde{R}_{\mathrm{m}}\right) K_{0}\left(-\mathrm{i} q \tilde{R}_{\mathrm{p}}\right)-J_{0}\left(q \tilde{R}_{\mathrm{p}}\right) K_{0}\left(-\mathrm{i} q \tilde{R}_{\mathrm{m}}\right)\right) E \\
-\left(\mathrm{i} J_{0}\left(q \tilde{R}_{\mathrm{p}}\right) K_{1}\left(-\mathrm{i} q \tilde{R}_{\mathrm{m}}\right)+J_{1}\left(q \tilde{R}_{\mathrm{m}}\right) K_{0}\left(-\mathrm{i} q \tilde{R}_{\mathrm{p}}\right)\right) q
\end{aligned}
$$

and

$$
q=\sqrt{-\frac{\mathrm{i} \tilde{\omega} \mu^{2}}{\varepsilon_{*}^{2} \tilde{D}_{\mathrm{N}}}}, \quad E=\frac{\exp \tilde{\eta}^{0}}{\varepsilon \varepsilon_{*}^{2} \tilde{D}_{\mathrm{N}}}
$$

Here, $J_{0}, J_{1}$ are the Bessel functions of the first kind, and $K_{0}, K_{1}$ are the modified Bessel functions of the second kind. Therefore, the problem for perturbation amplitudes is reduced to the system of two eqn (25) and (26), with the coefficient functions given by eqn (49), (21) and (27).

\subsection{Cathode side impedance}

To solve the system (25) and (26) we need the amplitude of oxygen concentration perturbation at the CCL/GDL interface, $\tilde{c}_{\mathrm{b}}^{1}(1)$, which appears in the boundary condition to eqn (26). This amplitude is given by ${ }^{\mathbf{1 7}}$

$$
\tilde{c}_{\mathrm{b}}^{1}(1)=-\left.\frac{\tan \left(\mu \tilde{l}_{\mathrm{b}} \sqrt{-\mathrm{i} \Omega / \tilde{D}_{\mathrm{b}}}\right)}{\mu \sqrt{-\mathrm{i} \Omega \tilde{D}_{\mathrm{b}}}} \tilde{D}_{\mathrm{p}} \frac{\partial \tilde{c}^{1}}{\partial \tilde{x}}\right|_{\tilde{x}=1}+\frac{\tilde{c}_{\mathrm{h}}^{1}}{\cos \left(\mu \tilde{l}_{\mathrm{b}} \sqrt{-\mathrm{i} \Omega / \tilde{D}_{\mathrm{b}}}\right)}
$$

where $\Omega$ is the reduced frequency

$$
\Omega=\frac{\tilde{\omega}}{\varepsilon_{*}^{2}}=\frac{\omega C_{\mathrm{dl}} l_{\mathrm{t}}^{2}}{\sigma_{\mathrm{N}}}
$$

$\tilde{D}_{\mathrm{b}}$ is the dimensionless oxygen diffusion coefficient in the GDL of a thickness $l_{\mathrm{b}}$ :

$$
\tilde{D}_{\mathrm{b}}=\frac{4 F D_{\mathrm{b}} c_{\mathrm{h}}^{\mathrm{in}}}{\sigma_{\mathrm{N}} b}, \quad \tilde{l}_{\mathrm{b}}=\frac{l_{\mathrm{b}}}{l_{\mathrm{t}}}
$$

and $\tilde{c}_{\mathrm{h}}^{1}$ is the amplitude of the oxygen concentration perturbation in channel. The latter value is obtained from the Fouriertransformed oxygen transport equation in the channel (ref. 18, chapter 6)

$$
\begin{aligned}
\lambda \tilde{J} \frac{\partial \tilde{c}_{\mathrm{h}}^{1}}{\partial \tilde{z}}= & \left(\mu \sqrt{-\mathrm{i} \Omega \tilde{D}_{\mathrm{b}}} \tan \left(\mu \tilde{l}_{\mathrm{b}} \sqrt{-\mathrm{i} \Omega / \tilde{D}_{\mathrm{b}}}\right)-\mathrm{i} \xi^{2} \varepsilon_{*}^{2} \Omega\right) \tilde{c}_{\mathrm{h}}^{1} \\
& -\frac{\tilde{D}_{\mathrm{p}} \partial \tilde{c}^{1} /\left.\partial \tilde{x}\right|_{\tilde{x}=1}}{\cos \left(\mu \tilde{l}_{\mathrm{b}} \sqrt{-\mathrm{i} \Omega / \tilde{D}_{\mathrm{b}}}\right)}, \\
\tilde{c}_{\mathrm{h}}^{1}(0)= & 0
\end{aligned}
$$

Here, $\tilde{z}=z / L$ is the dimensionless distance along the channel, $\tilde{J}$ $=J l_{\mathrm{t}} /\left(\sigma_{\mathrm{N}} b\right)$ is the dimensionless mean current density in the cell,

$$
\lambda=\frac{4 F h v c_{\mathrm{h}}^{\text {in }}}{L J}
$$

is the oxygen (air) flow stoichiometry, $v$ is the flow velocity in the channel, $h$ is the cathode channel depth, $L$ is the channel length, and $\xi$ is the dimensionless parameter

$$
\xi=\sqrt{\frac{4 F h c_{\mathrm{h}}^{\text {in }} l_{\mathrm{t}} i_{*}}{C_{\mathrm{dl}} \sigma_{\mathrm{N}} b^{2}}}
$$

\subsection{Impedance calculations and numerical details}

The whole problem is, thus, reduced to the core system (25), (26) with the Robin-type right boundary condition for $\tilde{c}^{1}$, which follows from eqn (32):

$$
\tilde{c}^{1}(1)+\left.\frac{\tan \left(\mu \tilde{l}_{\mathrm{b}} \sqrt{-\mathrm{i} \Omega / \tilde{D}_{\mathrm{b}}}\right)}{\mu \sqrt{-\mathrm{i} \Omega \tilde{D}_{\mathrm{b}}}} \tilde{D}_{\mathrm{p}} \frac{\partial \tilde{c}^{1}}{\partial \tilde{x}}\right|_{\tilde{x}=1}=\frac{\tilde{c}_{\mathrm{h}}^{1}}{\cos \left(\mu \tilde{l}_{\mathrm{b}} \sqrt{-\mathrm{i} \Omega / \tilde{D}_{\mathrm{b}}}\right)}
$$

The coefficient functions in eqn (25) and (26) are given by eqn (49), (21) and (27). Eqn (38) contains $\tilde{c}_{\mathrm{h}}^{1}(\tilde{z})$, which is determined 
from eqn (35). Note that iterations are required, as eqn (35) contains the derivative $\partial \tilde{c}^{1} / \partial \tilde{x}$; for detailed discussion of this model see (ref. 18, chapter 6). Finally, the system impedance is calculated according to

$$
\tilde{Z}=\left.\frac{\tilde{\eta}^{1}}{\partial \tilde{\eta}^{1} / \partial \tilde{x}}\right|_{\tilde{x}=0}
$$

The complex eqn (25), (26) and (35) have been transformed to equivalent system of real equations for the real and imaginary parts of $\tilde{\eta}^{1}, \tilde{c}^{1}$ and $\tilde{c}_{\mathrm{h}}^{1}$. The problem has been solved numerically in Python environment using the boundaryvalue and Cauchy problem solvers from the Python library SciPy. Following the quasi-2D approach, ${ }^{\mathbf{1 9}}$ the cell has been separated into $N$ virtual segments; solution to eqn (35) provided the channel value $\tilde{c}_{\mathrm{h}}^{1}(\tilde{z})$ for each segment. The local impedance of the $n^{\text {th }}$ segment $\tilde{Z}_{\text {seg, } n}$ has been calculated according to eqn (39); the total cell impedance $\tilde{Z}_{\text {cell }}$ has been obtained from

$$
\frac{1}{\tilde{Z}_{\text {cell }}}=\frac{1}{N} \sum_{n=1}^{N} \frac{1}{\tilde{Z}_{\text {seg }, n}}
$$

where $N=10$. An important element of cell impedance is "external" capacitance $C_{\text {ext. }}{ }^{20}$ Due to impurities, water in the CCL is a weak electrolyte, and $C_{\text {ext }}$ represents seemingly the carbon/water DL capacitance as a separate element connected in parallel to the cell impedance $Z_{\text {cell }}$, eqn (40). Incorporation of $C_{\text {ext }}$ greatly improves the quality of spectra fitting in the highfrequency region, and it leads to realistic values of the catalyst layer proton conductivity. ${ }^{20}$ The total cell impedance $Z_{\text {tot }}$ has thus been calculated from equation

$$
\frac{1}{Z_{\mathrm{tot}}}=\frac{1}{Z_{\mathrm{cell}}}+\mathrm{i} \omega C_{\mathrm{ext}} l_{\mathrm{t}}
$$

The model impedance has been fitted to the experimental impedance of the whole cell using the least_squares routine and the $\operatorname{trf}$ method from the SciPy. ${ }^{21}$ The merit function to be minimized is

$$
\sum_{m=1}^{M}\left(\operatorname{Re}\left(Z_{\mathrm{tot}, m}\right)-\operatorname{Re}\left(Z_{\text {exp }, m}\right)\right)^{2}+\left(\operatorname{Im}\left(Z_{\mathrm{tot}, m}\right)-\operatorname{Im}\left(Z_{\exp , m}\right)\right)^{2}
$$

where $Z_{\text {exp }}$ is the measured impedance, and index $m$ enumerates experimental frequency points. The $\operatorname{trf}$ algorithm implements a constrained method of minimization; description of this method states that "The method ... efficiently explore the whole space of variables." ${ }^{21}$ An alternative Levenberg-Marquardt (LM) algorithm works faster; however, being not constrained, in some cases LM returns physically unrealistic parameters.

Eight cell parameters have been declared as the fitting ones: $b, C_{\mathrm{dl}}, \sigma_{\mathrm{N}}, D_{\mathrm{p}}, D_{\mathrm{b}}, D_{\mathrm{N}}, l_{\mathrm{N}}$ and $C_{\text {ext }}$. Fitting has been performed assuming that in all the segments these parameters are the same, i.e., possible variation of parameters along the channel coordinate has been neglected.

\section{Results and discussion}

Fig. 2 shows the model Nafion film impedance $Z_{\mathrm{N}}$ calculated for the cell current density of $50 \mathrm{~mA} \mathrm{~cm} \mathrm{~cm}^{-2}$ and the parameters indicated in figure caption and in Table 1 . The film impedance has been separated as following: first, the complete cell impedance with the parameters from Table 1 has been calculated. Second, the impedance with $K_{\mathrm{H}}=1$ and infinite oxygen diffusion coefficient in the Nafion film has been calculated. Subtracting the second impedance from the first one gives the "pure" Nafion film impedance (Fig. 2).

As can be seen, for the CCL thickness of $3 \mu \mathrm{m}$, the characteristic (summit) frequency $f_{*}$ of $Z_{\mathrm{N}}$ (corresponding to the peak of $-\operatorname{Im}\left(Z_{\mathrm{N}}\right)$ ) is about $12 \mathrm{~Hz}$ (Fig. 2b), which is a rather surprising result. Indeed, Nafion film may be thought of as a finite-length transport layer connected to the Pt surface. Warburg solution gives for the characteristic angular frequency of this layer $\omega_{*}=$ $2.54 D_{\mathrm{N}} / l_{\mathrm{N}}{ }^{2}$. With the parameters from Table 1 and Fig. 2 caption we get $\omega_{*} \simeq 2.54 \times 10^{6} \mathrm{~s}^{-1}$, or $f_{*} \simeq 600 \mathrm{kHz}$. This value is nearly two orders of magnitude higher than the maximal frequency usually used in impedance spectroscopy of fuel cells.

In fact, the pore and Nafion film form a unified, two-layer transport system for oxygen molecules. The characteristic frequency of $\mathrm{O}_{2}$ transport in this system is determined by the slowest part of the pathway, which is transport in the void pore. Calculation of $Z_{\mathrm{N}}$ with twice larger CCL thickness $(6 \mu \mathrm{m})$
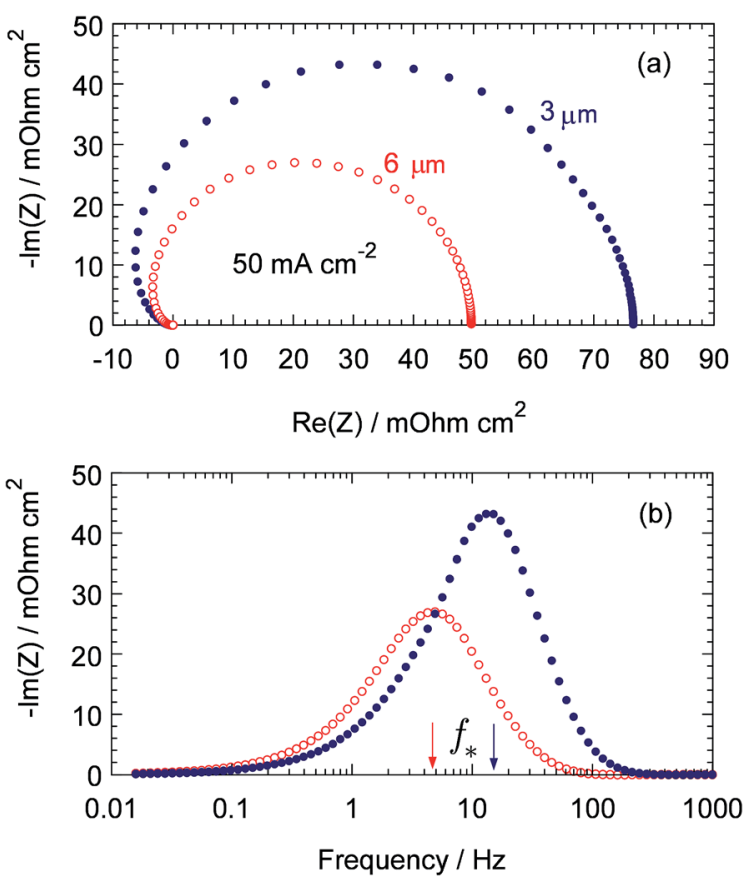

Fig. 2 (a) Model Nyquist spectrum of the Nafion film impedance, (b) Frequency dependence of the imaginary part of impedance. Parameters for calculations: $b=0.025 \mathrm{~V}, C_{\mathrm{dl}}=25 \mathrm{~F} \mathrm{~cm}^{-3}, \sigma_{\mathrm{N}}=3 \times 10^{-3} \mathrm{~S}$ $\mathrm{cm}^{-1}, D_{\mathrm{p}}=5 \times 10^{-6} \mathrm{~cm}^{2} \mathrm{~s}^{-1}, D_{\mathrm{N}}=1 \times 10^{-6} \mathrm{~cm}^{2} \mathrm{~s}^{-1}, D_{\mathrm{b}}=0.18 \mathrm{~cm}^{2}$ $\mathrm{s}^{-1}$. Points: the CCL thickness $l_{\mathrm{t}}=3 \mu \mathrm{m}$, open circles: $l_{\mathrm{t}}=6 \mu \mathrm{m}$. 
Table 1 Cell geometrical and operating parameters. A/C stands for anode/cathode
Catalyst loading A/C, $\mathrm{mg} \mathrm{cm}^{-2}$

CCL thickness (pore length) $l_{\mathrm{t}}, \mathrm{cm}$

Nafion film thickness $l_{\mathrm{N}}, \mathrm{cm}$ (assumed)

GDL thickness $l_{\mathrm{b}}, \mathrm{cm}$

Open pore radius $R_{\mathrm{p}}$, cm (assumed)

Channel depth $h$, $\mathrm{cm}$

Channel length $L, \mathrm{~cm}$

ORR exchange current density $i_{*}, \mathrm{~A} \mathrm{~cm}^{-3}$ (assumed)

Henry's constant for oxygen solubility in water at $80 \quad 6.76 \times 10^{-3}$

${ }^{\circ} \mathrm{C}, \mathrm{mol} \mathrm{mol}^{-1}$

Flow stoichiometry A/C

Relative humidity A/C

Cathode absolute pressure, $\mathrm{kPa}$

Cell temperature, $\mathrm{K}$

$2 / 4$

$100 \% / 50 \%$

150

$273+80$
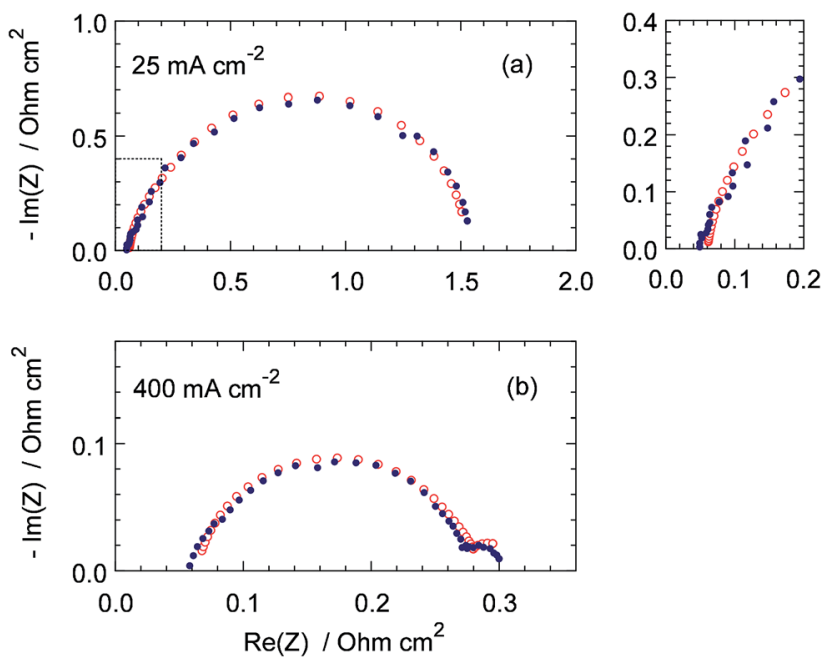

Fig. 4 Experimental (points) and fitted model (open circles) Nyquist plots of the cell impedance for the indicated current densities. The model and experimental points are shown for the same frequencies.

Note that the spectra in Fig. 4 do not exhibit $45^{\circ}$ straight line in the high-frequency region (Fig. 4a, right panel). Typical modern PEMFCs exhibit high slope of Nyquist impedance spectra in the HF region; this slope greatly exceeds following from theory $45^{\circ}$ (ref. 23 ). There have been a lot of discussions in literature on the origin of this effect. Recently, we have suggested that the large slope is due to "external" capacitance $C_{\text {ext }}$ connected in parallel to our model impedance (see also discussion above). ${ }^{20}$

The parameters resulted from fitting are shown in Fig. 6. Of largest interest is the oxygen diffusion coefficients in the Nafion film $D_{\mathrm{N}}$ (Fig. 6a) and the Nafion film thickness (Fig. 6b). As can
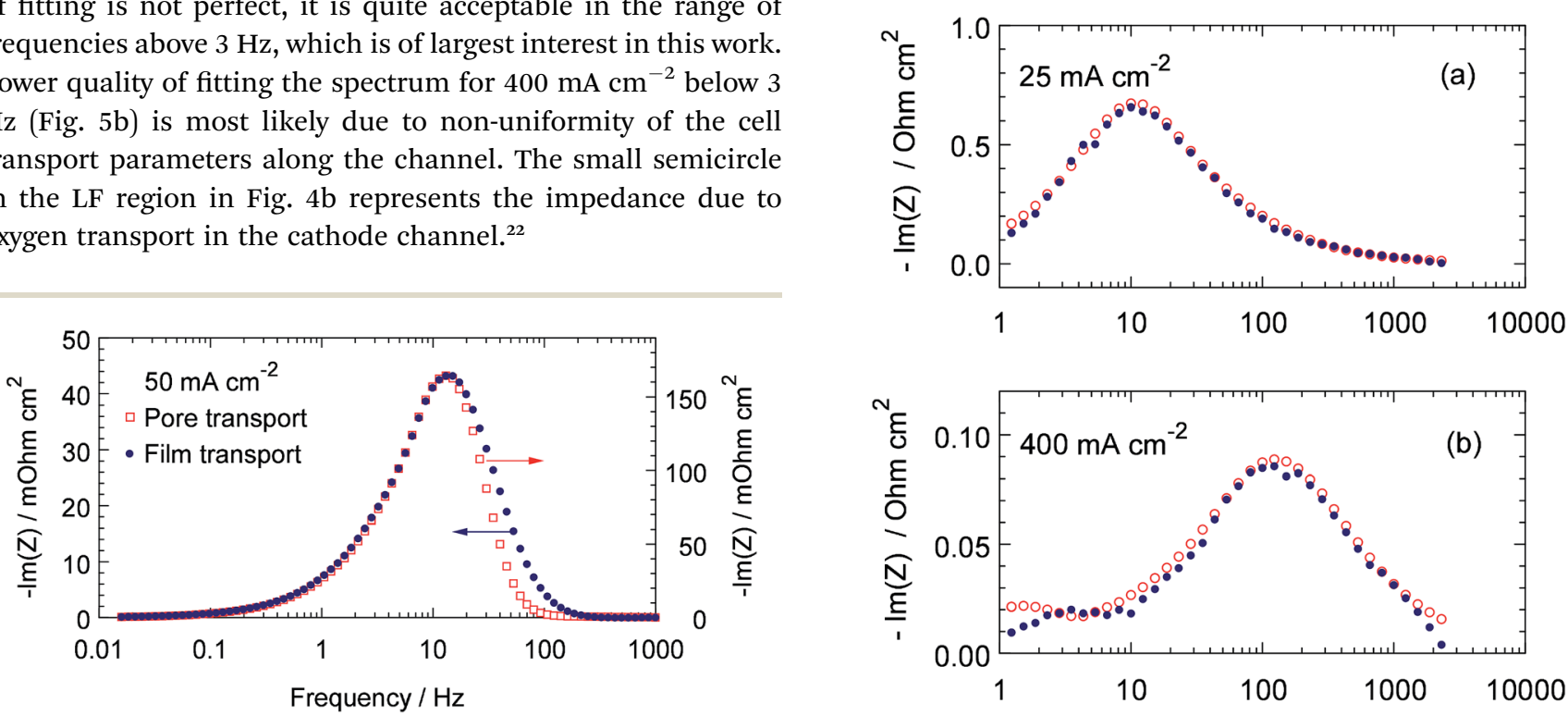

Fig. 3 Frequency dependencies of the imaginary part of impedance of the oxygen transport in the Nafion film (points) and in the pore volume (open squares). Parameters for calculations are the same as in Fig. 2.

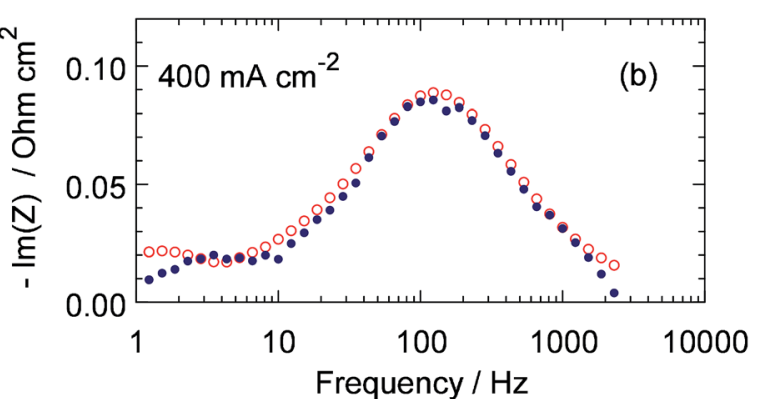

Fig. 5 Experimental (points) and fitted model (open circles) frequency dependencies of $\operatorname{Im}(Z)$ for the same spectra as in Fig. 4. 

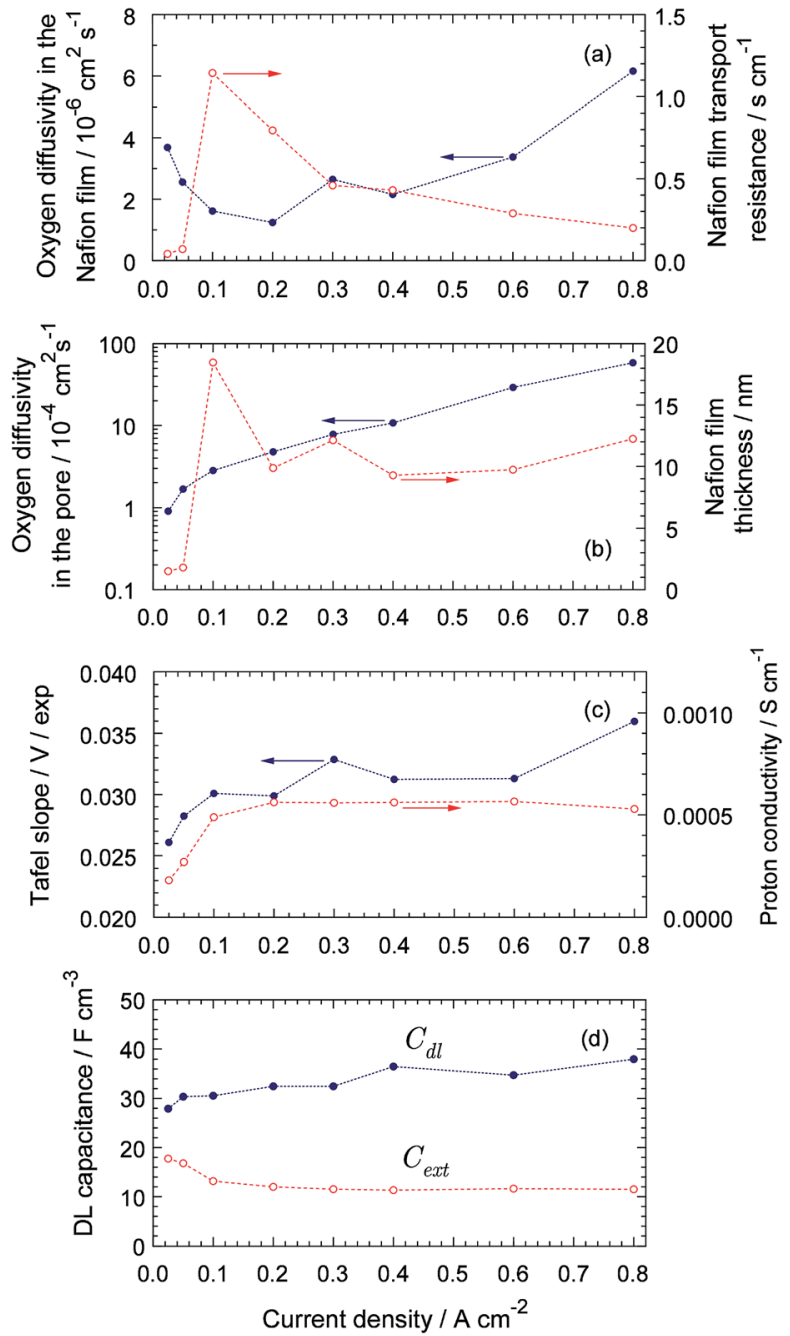

Fig. 6 Cell parameters resulted from spectra fitting vs. cell current density. (a) Oxygen diffusivity in the Nafion film and the film transport resistivity. (b) Oxygen diffusivity in the pore volume and the Nafion film thickness, (c) ORR Tafel slope per exponential basis and Nafion film proton conductivity, (d) Double layer capacitance $C_{\mathrm{dl}}$ and "external" capacitance $C_{\text {ext }}$.

be seen, $D_{\mathrm{N}}$ varies in the range $1 \times 10^{-6} \mathrm{~cm}^{2} \mathrm{~s}^{-1}$ to $\simeq 6 \times 10^{-6}$ $\mathrm{cm}^{2} \mathrm{~s}^{-1}$ (Fig. 6a). The lowest value of $D_{\mathrm{N}}$ agrees well with the oxygen diffusivity in the bulk Nafion 117 , which is $0.96 \times 10^{-6}$ $\mathrm{cm}^{2} \mathrm{~s}^{-1}$ (ref. 24). The reason for $D_{\mathrm{N}}$ variation with the cell current is not clear yet; perhaps, this variation is due to change of the film surface properties. ${ }^{6}$ On the other hand, the oxygen transport model above is essentially a two-scale model, with the large-scale through-plane oxygen transport modeled as a transport in a straight pore, and the small-scale transport to the $\mathrm{Pt} / \mathrm{C}$ surface perpendicular to the pore. In Fig. 1, the small-scale transport path is represented by the Nafion film; however, part of this path could include transport through the primary nanopores. The transport properties of these pores could vary with the cell current, which may contribute to the variation of effective $D_{\mathrm{N}}$ with current density.
Table 2 Oxygen transport resistivities in the low-Pt PEMFC resulted from fitting the spectrum for $200 \mathrm{~mA} \mathrm{~cm}^{-2}$

\begin{tabular}{ll}
\hline Oxygen transport & $\begin{array}{l}\text { Differential resistivity, } \\
\mathrm{m} \Omega \mathrm{cm}^{2}\end{array}$ \\
\hline In the Nafion film & 48.7 \\
In the GDL & 21.9 \\
In the channel & 11.9 \\
In the void pore & 7.07
\end{tabular}

Excluding the low-current points, the Nafion film thickness varies in the range of 10 to $13 \mathrm{~nm}$ without any distinct correlation with the current density (Fig. 6b); this thickness agrees well with the literature data. ${ }^{25,26}$ The points for 25, 50 and 100 $\mathrm{mA} \mathrm{cm}^{-2}$ on this plot could be not accurate, as at small currents, the contribution of oxygen transport in Nafion film to $Z_{\text {tot }}$ is small and the model may not be able to capture it. The transport resistivity of the film $R_{\mathrm{N}}=l_{\mathrm{N}} / D_{\mathrm{N}}$ decays from $0.8 \mathrm{~s}$ $\mathrm{cm}^{-1}$ at $200 \mathrm{~mA} \mathrm{~cm}{ }^{-2}$ down to $0.2 \mathrm{~s} \mathrm{~cm}^{-1}$ at $800 \mathrm{~mA} \mathrm{~cm}$ (Fig. 6a).

The ORR Tafel slope exhibits variation in the range of 26 to $35 \mathrm{mV}$ per exponential basis, around the average value of $\simeq 30$ $\mathrm{mV}$ (Fig. 6c), which translates to typical for Pt/C systems value of $70 \mathrm{mV}$ per decade. ${ }^{27}$ The Pt/Nafion double layer capacitance grows from 30 to $40 \mathrm{~F} \mathrm{~cm}^{-3}$ (Fig. 6d). This growth seemingly is due to cleaning of $\mathrm{Pt}$ surface from oxides at lower cell potentials. ${ }^{28}$

The CCL proton conductivity is nearly constant at $6 \times 10^{-4} \mathrm{~S}$ $\mathrm{cm}^{-1}$ (Fig. 6c). The oxygen diffusion coefficient in the pore volume increases by two orders of magnitude, from $10^{-4}$ to $\simeq$ $10^{-2} \mathrm{~cm}^{2} \mathrm{~s}^{-1}$ (Fig. 6b). The origin of this growth yet is unknown; possible explanation would be formation of a pressure gradient pushing liquid water out of the CCL. The low-current value of $D_{\mathrm{p}}$ suggests that the low-Pt CCL is partially flooded. Indeed, the oxygen diffusion coefficient in pure water at $80^{\circ} \mathrm{C}$ is $0.53 \times 10^{-4}$ $\mathrm{cm}^{2} \mathrm{~s}^{-1}$ (ref. 29) and the low-current part of the curve in Fig. $6 \mathrm{~d}$ is close to this value.

Table 2 shows the contributions of various transport processes to the total differential resistivity of the cell at the current density of $200 \mathrm{~mA} \mathrm{~cm}{ }^{-2}$. As can be seen, already at quite moderate current density, among all the oxygen transport processes on the cathode side, Nafion film gives the largest contribution to the voltage loss.

\section{Conclusions}

We report a model for PEM fuel cell impedance taking into account oxygen transport through the Nafion film covering $\mathrm{Pt} / \mathrm{C}$ agglomerates. The cathode catalyst layer is modeled by a cylindrical straight pore penetrating through the CCL thickness. The pore is separated from a coaxial $\mathrm{Pt} / \mathrm{C}$ cylinder by the thin Nafion film. The impedance model is based on the oxygen diffusion equations along the void pore and through the Nafion film, coupled by the Tafel equation for the ORR rate at the film/metal interface. In the limit of zero Nafion film thickness the model reduces to the standard macrohomogeneous model for the CCL 
performance. The numerical impedance model is fitted to experimental impedance spectra of a low-Pt PEM fuel cell with the cathode Pt loading of $0.1 \mathrm{mg}_{\mathrm{Pt}} \mathrm{cm}^{-2}$. Fitting gives the oxygen diffusion coefficient in the film increasing from $1.5 \times 10^{-6}$ to $6 \times$ $10^{-6} \mathrm{~cm}^{2} \mathrm{~s}^{-1}$ as the cell current increases from 200 to $800 \mathrm{~mA}$ $\mathrm{cm}^{-2}$. The low-current value of $1.5 \times 10^{-6} \mathrm{~cm}^{2} \mathrm{~s}^{-1}$ by the order of magnitude agrees well with the oxygen diffusivity in the bulk Nafion 117. The Nafion film thickness varies in the range of 10 to $13 \mathrm{~nm}$ showing no correlation with the cell current. Overall, the oxygen transport resistivity of the film decreases from $\simeq 1$ to $0.2 \mathrm{~s}$ $\mathrm{cm}^{-1}$ with the growth of the cell current density. Last but not least, fitting returns low value of the through-plane oxygen diffusivity in the CCL, which indicates that the low-Pt electrode is partly flooded.

\section{Appendix}

\section{Derivation of static equations}

Th system of equations for $\tilde{c}^{0}, \tilde{c}_{\mathrm{N}}^{0}$ and $\tilde{\eta}^{0}$ is obtained from eqn (12)-(14) by chalking out the time derivatives:

$$
\begin{gathered}
\tilde{D}_{\mathrm{p}} \frac{\partial^{2} \tilde{c}^{0}}{\partial \tilde{x}^{2}}=-\varepsilon \tilde{N}_{\mathrm{N}, \mathrm{m}}^{0},\left.\quad \frac{\partial \tilde{c}^{0}}{\partial \tilde{x}}\right|_{\tilde{x}=0}=0, \quad \tilde{c}^{0}(1)=\tilde{c}_{1}, \\
\frac{1}{\tilde{r}} \frac{\partial}{\partial \tilde{r}}\left(\tilde{r} \frac{\partial \tilde{c}_{\mathrm{N}}^{0}}{\partial \tilde{r}}\right)=0, \quad \tilde{c}_{\mathrm{N}, \mathrm{p}}^{0}=K_{\mathrm{H}} \tilde{c}^{0}(\tilde{x}), \\
\left.\varepsilon \varepsilon_{*}^{2} \tilde{D}_{\mathrm{N}} \frac{\partial \tilde{c}_{\mathrm{N}}^{0}}{\partial \tilde{r}}\right|_{\tilde{r}=\tilde{R}_{\mathrm{m}}}=-\tilde{c}_{\mathrm{N}, \mathrm{m}}^{0} \exp \tilde{\eta}^{0} \\
\varepsilon_{*}^{2} \frac{\partial^{2} \tilde{\eta}^{0}}{\partial \tilde{x}^{2}}=\tilde{c}_{\mathrm{N}, \mathrm{m}}^{0} \exp \tilde{\eta}^{0}, \quad \tilde{\eta}^{0}(0)=\tilde{\eta}_{0},\left.\quad \frac{\partial \tilde{\eta}^{0}}{\partial \tilde{x}}\right|_{\tilde{x}=1}=0
\end{gathered}
$$

where the superscript 0 marks the steady-state variables.

Unfortunately, the total static overpotential $\tilde{\eta}_{0}$ appearing in eqn (45) is unknown; calculation of this parameters requires iterations. The impedance measurements are usually done at constant current density in the cell $\tilde{j}_{0}$, and to avoid iterations, the static electric problem has to be reformulated in terms of the proton current density $\tilde{j}(\tilde{x})$. Consider first the static version of eqn (7); in the dimensionless variables this equation reads

$$
\varepsilon_{*}^{2} \frac{\partial \hat{j}^{0}}{\partial \tilde{x}}=-\tilde{c}_{\mathrm{N}, \mathrm{m}}^{0} \exp \tilde{\eta}^{0}
$$

Here, $\tilde{c}_{\mathrm{N}, \mathrm{m}}^{0}$ depends parametrically of $\tilde{x}$ (see below).

To derive equation for $\tilde{j}^{0}$ equivalent to eqn (45), we differentiate eqn (46) over $\tilde{x}$, which gives

$$
\varepsilon_{*}^{2} \frac{\partial^{2} \tilde{j}^{0}}{\partial \tilde{x}^{2}}=-\exp \left(\tilde{\eta}^{0}\right)\left(\frac{\partial \tilde{c}_{\mathrm{N}, \mathrm{m}}^{0}}{\partial \tilde{x}}+\tilde{c}_{\mathrm{N}, \mathrm{m}}^{0} \frac{\partial \tilde{\eta}^{0}}{\partial \tilde{x}}\right)
$$

Substituting here exp $\tilde{\eta}^{0}$ from eqn (46) and using the Ohm's law $\tilde{j}^{0}=-\partial \tilde{\eta}^{0} / \partial \tilde{x}$, after simple algebra we come to

$$
\frac{\partial^{2} \tilde{j}^{0}}{\partial \tilde{x}^{2}}-\left(\frac{\partial \ln \left(\tilde{c}_{\mathrm{N}, \mathrm{m}}^{0}\right)}{\partial \tilde{x}}-\tilde{j}^{0}\right) \frac{\partial \tilde{j}^{0}}{\partial \tilde{x}}=0 .
$$

$$
\tilde{c}_{\mathrm{N}}^{0}=\left(\frac{\tilde{R}_{\mathrm{m}} \ln \left(\tilde{R}_{\mathrm{m}} / \tilde{r}\right) \exp \tilde{\eta}^{0}+\varepsilon \varepsilon_{*}^{2} \tilde{D}_{\mathrm{N}}}{\tilde{R}_{\mathrm{m}} \ln \left(\tilde{R}_{\mathrm{m}} / \tilde{R}_{\mathrm{p}}\right) \exp \tilde{\eta}^{0}+\varepsilon \varepsilon_{*}^{2} \tilde{D}_{\mathrm{N}}}\right) K_{\mathrm{H}} \tilde{c}^{0}
$$

Setting $\tilde{r}=\tilde{R}_{\mathrm{m}}$ in this solution, we obtain

$$
\tilde{c}_{\mathrm{N}, \mathrm{m}}^{0}=\frac{K_{\mathrm{H}} \tilde{c}^{0}}{1+\alpha \exp \tilde{\eta}^{0}}
$$

where $\alpha$ is given by eqn (20). With this, eqn (46) takes the form

$$
\varepsilon_{*}^{2} \frac{\partial \tilde{j}^{0}}{\partial \tilde{x}}=-\frac{K_{\mathrm{H}} \tilde{c}^{0} \exp \tilde{\eta}^{0}}{1+\alpha \exp \tilde{\eta}^{0}}
$$

Solving eqn (50) for $\exp \tilde{\eta}^{0}$ we find

$$
\exp \tilde{\eta}^{0}=-\frac{\varepsilon_{*}^{2} \partial \hat{j}^{0} / \partial \tilde{x}}{K_{\mathrm{H}} \tilde{c}^{0}+\alpha \varepsilon_{*}^{2} \partial \hat{j}^{0} / \partial \tilde{x}}
$$

Calculating $\partial \ln \left(\tilde{c}_{\mathrm{N}, \mathrm{m}}^{0}\right) / \partial \tilde{x}$ with eqn (49), we get

$$
\frac{\partial \ln \left(\tilde{c}_{\mathrm{N}, \mathrm{m}}^{0}\right)}{\partial \tilde{x}}=\frac{\partial \ln \left(\tilde{c}^{0}\right)}{\partial \tilde{x}}-\left(\frac{\alpha \exp \tilde{\eta}^{0}}{1+\alpha \exp \tilde{\eta}^{0}}\right) \frac{\partial \tilde{\eta}^{0}}{\partial \tilde{x}}
$$

Taking into account the Ohm's law $\partial \tilde{\eta}^{0} / \partial \tilde{x}=-\tilde{j}^{0}$ and eqn (51), we get

$$
\frac{\partial \ln \left(\tilde{c}_{\mathrm{N}, \mathrm{m}}^{0}\right)}{\partial \tilde{x}}=\frac{\partial \ln \left(\tilde{c}^{0}\right)}{\partial \tilde{x}}-\left(\frac{\alpha \varepsilon_{*}^{2}}{K_{\mathrm{H}} \tilde{c}^{0}}\right) \hat{j}^{0} \frac{\partial \hat{j}^{0}}{\partial \tilde{x}}
$$

Using eqn (53) in eqn (47) we finally get a problem for $\tilde{j}^{0}$, eqn (18). A great advantage of the problem (18) is that the boundary conditions are known and iterations are not required.

To find equation for the static oxygen concentration in the pore volume $\tilde{c}^{0}$, we note that eqn (48) allows us to calculate the flux $\tilde{N}_{\mathrm{N}, \mathrm{m}}^{0}$, which appears in eqn (43). Calculating derivative $\partial \tilde{c}_{\mathrm{N}}^{0} / \partial \tilde{r}$, multiplying the result by $\tilde{D}_{\mathrm{N}}$ and setting $\tilde{r}=$ $\tilde{R}_{\mathrm{m}}$, we get

$$
\tilde{N}_{\mathrm{N}, \mathrm{m}}^{0}=-\frac{K_{\mathrm{H}} \tilde{c}^{0} \exp \tilde{\eta}^{0}}{\varepsilon \varepsilon_{*}^{2}\left(1+\alpha \exp \tilde{\eta}^{0}\right)}
$$

Using here eqn (51), we come to

$$
\tilde{N}_{\mathrm{N}, \mathrm{m}}^{0}=\frac{1}{\varepsilon} \frac{\partial j^{0}}{\partial \tilde{x}}
$$

With this, eqn (43) transforms to

$$
\tilde{D}_{\mathrm{p}} \frac{\partial^{2} \tilde{c}^{0}}{\partial \tilde{x}^{2}}=-\frac{\partial \tilde{j}^{0}}{\partial \tilde{x}},\left.\quad \frac{\partial \tilde{c}^{0}}{\partial \tilde{x}}\right|_{\tilde{x}=0}=0, \quad \tilde{c}^{0}(1)=\tilde{c}_{1},
$$

Integrating this equation once, we come to eqn (19), which is a standard MHM equation for oxygen transport in the CCL. ${ }^{30}$

Thus, the static problem is reduced to the system of eqn (18) and (19). With $\tilde{c}^{0}(\tilde{x})$ and $\tilde{j}^{0}(\tilde{x})$, the static overpotential $\tilde{\eta}^{0}$ is obtained from eqn (51), which leads to eqn (21). If necessary, the radial shape of dissolved oxygen concentration $\tilde{c}_{\mathrm{N}}^{0}$ can be calculated from eqn (48).

To find $\tilde{c}_{\mathrm{N}, \mathrm{m}}^{0}$ we solve eqn (44): 


\section{Conflicts of interest}

There are no conflicts to declare.

\section{Nomenclature}

Marks dimensionless variables

$b$

$C_{\mathrm{dl}}$

$C_{\text {ext }}$

$c$

$c_{\mathrm{b}}$

$c_{\mathrm{h}}$

$c_{\mathrm{N}}$

$c_{\mathrm{h}}^{\mathrm{in}}$

$D_{\mathrm{p}}$

$D_{\mathrm{N}}$

F

$i_{*}$

i

j

$j_{0}$

$K_{\mathrm{H}}$

$l_{\mathrm{t}}$

$l_{\mathrm{N}}$

$N_{\mathrm{N}}$

$R_{\mathrm{m}}$

$R_{\mathrm{p}}$

$r$

$v$

$x$

$z$

\section{Subscripts}

$\begin{array}{ll}0 & \text { Membrane/CCL interface } \\ 1 & \text { CCL/GDL interface } \\ \mathrm{t} & \text { Catalyst layer }\end{array}$

\section{Superscripts}

0

1

Steady-state value

Small-amplitude perturbation

\section{Greek}

$\alpha \quad$ Dimensionless parameter, eqn (20)

$\varepsilon_{*} \quad$ Dimensionless Newman's reaction penetration depth, eqn (17)

$\begin{array}{ll}\varepsilon & \text { Dimensionless parameter, eqn (17) } \\ \eta & \text { ORR overpotential, positive by convention, } \mathrm{V} \\ \lambda & \text { Air flow stoichiometry, eqn (36) } \\ \xi & \text { Dimensionless parameter, eqn (37) } \\ \sigma_{\mathrm{N}} & \text { Nafion film proton conductivity, } \mathrm{S} \mathrm{cm}^{-1} \\ \Omega & \text { Dimensionless reduced angular frequency of the AC } \\ & \text { signal, eqn (33) } \\ \omega & \text { Angular frequency of the AC signal, } \mathrm{s}^{-1}\end{array}$

\section{Acknowledgements}

T. Reshetenko gratefully acknowledges funding from US Office of Naval Research (N00014-18-1-2127) and US Army Research Office (W911NF-15-1-0188). The authors are thankful to Günter Randolf and Jack Huizingh for valuable help with the system operation, and to the Hawaiian Electric Company for ongoing support of the Hawaii Sustainable Energy Research Facility.

\section{Notes and references}

1 T. Grezler, D. Gaulk and P. Sinha, J. Electrochem. Soc., 2012, 159, F831-F840.

2 A. Kongkanand and M. F. Mathias, J. Phys. Chem. Lett., 2016, 7, 1127-1137.

3 J. P. Owejan, J. E. Owejan and W. Gu, J. Electrochem. Soc., 2013, 160, F824-F833.

4 A. Z. Weber and A. Kusoglu, J. Mater. Chem. A, 2014, 2, 17207-17211.

5 M.-J. Choo, K.-H. Oh, J.-K. Park and H.-T. Kim, ChemElectroChem, 2015, 2, 382-388.

6 K. Kudo, R. Jinnouchi and Y. Morimoto, Electrochim. Acta, 2016, 209, 682-690.

7 A. Kongkanand, W. Gu and M. Mathias, Fuel Cells and Hydrogen production, Springer, New-York, 2018, pp. 323-342.

8 A. Kusoglu, Fuel Cells and Hydrogen production, Springer, New York, 2018, pp. 417-438.

9 T. Muzaffar, T. Kadyk and M. Eikerling, Sustainable Energy Fuels, 2018, 2, 1189-1196.

10 M. Eikerling, J. Electrochem. Soc., 2006, 153, E58-E70.

11 A. Kulikovsky, Electrochem. Commun., 2019, 103, 61-65.

12 W. Wei, W. Ye, J. Wang, C. Huang, J.-B. Xiong, H. Qiao, S. Cui, W. Chen, L. Mi and P. Yan, ACS Appl. Mater. Interfaces, 2019, 11, 32269-32281.

13 M. D. Murbach and D. T. Schwartz, J. Electrochem. Soc., 2018, 165, A297-A304.

14 T. V. Reshetenko, G. Bender, K. Bethune and R. Rocheleau, Electrochim. Acta, 2011, 56, 8700-8710.

15 T. Reshetenko and A. Kulikovsky, J. Electrochem. Soc., 2017, 164, F1633-F1640.

16 P. Dobson, C. Lei, T. Navessin and M. Secanell, J. Electrochem. Soc., 2012, 159, B514-B523.

17 T. Reshetenko and A. Kulikovsky, J. Electrochem. Soc., 2016, 163, F1100-F1106.

18 A. Kulikovsky, Analytical Models for PEM Fuel Cell Impedance, Self-publishing, Eisma, 2018, http://www.amazon.com/ Andrei-Kulikovsky/e/B00KBW7KVY. 
19 T. Reshetenko and A. Kulikovsky, J. Electrochem. Soc., 2018, 165, F291-F296.

20 T. Reshetenko and A. Kulikovsky, J. Electrochem. Soc., 2019, DOI: 10.1149/2.1201915.jes.

21 scipy.optimize.least_squares, 2018, https://docs.scipy.org/ $\mathrm{doc} / \mathrm{scipy} /$ reference/generated/ scipy.optimize.least_squares.html.

22 A. Kulikovsky and O. Shamardina, J. Electrochem. Soc., 2015, 162, F1068-F1077.

23 A. A. Kulikovsky and M. Eikerling, J. Electroanal. Chem., 2013, 691, 13-17.

24 V. A. Sethuraman, S. Khan, J. S. Jur, A. T. Haug and J. Weidner, Electrochim. Acta, 2009, 54, 6850-6860.
25 S. Holdcroft, Chem. Mater., 2014, 26, 383-393.

26 M. Lopez-Haro, L. Guétaz, T. Printemps, A. Morin, S. Escribano, P.-H. Jouneau, P. Bayle-Guillemaud, F. Chandezon and G. Gebel, Nat. Commun., 2014, 5, 5229.

27 Y. Liu, C. Ji, W. Gu, J. Jorne and H. A. Gasteiger, J. Electrochem. Soc., 2011, 158, B614-B621.

28 Y. Liu, M. Mathias and J. Zhang, Electrochem. Solid-State Lett., 2010, 13, B1-B3.

29 P. Han and D. M. Bartels, J. Chem. Phys., 1996, 100, 55975602.

30 M. Eikerling and A. A. Kornyshev, J. Electroanal. Chem., 1998, 453, 89-106. 